\title{
Dynamic Simulation of Modifiable Bipedal Walking on Uneven Terrain with Unknown Height
}

\author{
Young-Dae Hong* and Ki-Baek Lee ${ }^{\dagger}$
}

\begin{abstract}
To achieve bipedal walking in real human environments, a bipedal robot should be capable of modifiable walking both on uneven terrain with different heights and on flat terrain. In this paper, a novel walking pattern generator based on a 3-D linear inverted pendulum model (LIPM) is proposed to achieve this objective. By adopting a zero moment point (ZMP) variation scheme in real time, it is possible to change the center-of-mass (COM) position and the velocity of the 3-D LIPM throughout the single support phase. Consequently, the proposed method offers the ability to generate a modifiable pattern for walking on uneven terrain without the necessity for any extra footsteps to adjust the COM motion. In addition, a control strategy for bipedal walking on uneven terrain with unknown height is developed. The torques and ground reaction force are measured through forcesensing resisters (FSRs) on each foot and the foot of the robot is modeled as three virtual springdamper models for the disturbance compensation. The methods for generating the foot and vertical COM of 3-D LIPM trajectories are proposed to achieve modifiable bipedal walking on uneven terrain without any information regarding the height of the terrain. The effectiveness of the proposed method is confirmed through dynamic simulations.
\end{abstract}

Keywords: Bipedal robot, Humanoid robot, Modifiable walking pattern, Uneven terrain locomotion

\section{Introduction}

Stable bipedal walking is one of the key research topics in the field of robotics, and several approaches have been developed to generate walking patterns for bipedal robots [1-6]. These approaches have been developed under the assumption that the robot is walking on flat terrain. However, for bipedal walking in real human environments, which contain both flat and uneven terrains of different heights, it is necessary to be able to generate walking patterns for bipedal walking on uneven terrain.

For this purpose, it has been proposed that a bipedal robot can be approximated using a linear inverted pendulum model (LIPM) [7]. An attempt to utilize a sensor system comprising a body-inclination sensor, a joint encoder, and a force-sensing resister (FSR) for feedforward dynamic walking and sensory feedback has been reported [8]. Walking patterns have also been generated to allow for auxiliary zero moment point (ZMP) control using a preview controller [9], and to control the posture of the robot using an attitude measurement sensor and a center-of-pressure controller [10]. Moreover, approaches have been proposed to modify the landing timing and position of the swing leg using a ZMP criteria map [11] and to generate walking patterns using the contact wrench sum as a stability criterion [12]. In addition, an environment mode converted from the

$\dagger$ Corresponding Author: Dept. of Electrical Engineering, Kwangwoon University, Korea. (kblee@kw.ac.kr).

* Dept. of Electrical and Computer Engineering, Ajou University, Korea. (ydhong@ajou.ac.kr).

Received: March 5, 2015; Accepted: November 17, 2015 sensor information collected by the FSRs has been proposed to represent the contact state between the terrain and soles [13], and a foot mechanism to detect terrain height using an optical distance sensor has been developed [14]. However, the bipedal robots in the previous studies related to walking pattern generation on uneven terrain have been unable to independently modify the single and double support times, the sagittal and lateral step lengths, and the walking direction of the swing leg on uneven terrain at every footstep.

In this paper, for modifiable bipedal walking on both uneven terrain and flat terrain, a walking pattern generator based on the 3-D LIPM is utilized [15]. By adopting the ZMP variation scheme in real time, it is possible to consider changes in the COM position and the velocity in the 3-D LIPM throughout the single support phase. Consequently, the proposed method offers the ability to generate a modifiable pattern for walking on uneven terrain without the necessity for any extra footsteps to adjust the COM motion. In addition, a control strategy for bipedal walking on uneven terrain with unknown height is developed. The torques and ground reaction force are measured through the FSRs on each foot and the foot of the robot is modeled as three virtual spring-damper models for the disturbance compensation. The methods for generating the foot and vertical COM of 3-D LIPM trajectories are proposed to achieve modifiable bipedal walking on uneven terrain without any information regarding the height of the terrain.

This paper is organized as follows. Section 2 presents the modifiable walking pattern. The generation of the $\mathrm{COM}$ trajectory is described, and the conversion of the 
command state (CS) into the desired walking state (WS) is explained. In Section 3, the control strategy for bipedal walking on uneven terrain with an unknown height is proposed. The virtual spring-damper model that is used to compensate for disturbances is described, and the generation of the foot and vertical COM trajectories for bipedal walking on uneven terrain with an unknown height is explained. In Section 4, the simulation results are presented, and finally, conclusions follow in Section 5.

\section{Modifiable Walking Pattern}

\subsection{COM trajectory generation}

Bipedal walking consists of single and double support phases. In the single support phase, the primary dynamics of bipedal walking on flat terrain can be simplified to a 3-D LIPM, as illustrated in Fig. 1 [1]. In this 3-D LIPM, the mass is concentrated at a single point, and the support leg of the bipedal robot is treated as a weightless telescopic limb. In addition, the vertical motion of the mass is not considered. Consequently, it is possible to decouple the sagittal and lateral COM motion equations. The sagittal and lateral COM motions of the 3-D LIPM can be obtained from the dynamic equation of the 3-D LIPM as follows:

$$
\begin{aligned}
& \mathbf{x}_{f}=\mathbf{A}(t) \mathbf{x}_{i}+\mathbf{B}_{p}(t) \\
& \mathbf{y}_{f}=\mathbf{A}(t) \mathbf{y}_{i}+\mathbf{B}_{q}(t)
\end{aligned}
$$

with

$$
\begin{aligned}
& \mathbf{A}(t)=\left[\begin{array}{l}
c(t) s(t) \\
s(t) c(t)
\end{array}\right] \\
& \mathbf{B}_{p}(t)=-\frac{1}{T_{c}}\left[\begin{array}{l}
(s * p)(t) \\
(c * p)(t)
\end{array}\right], \quad \mathbf{B}_{q}(t)=-\frac{1}{T_{c}}\left[\begin{array}{l}
(s * q)(t) \\
(c * q)(t)
\end{array}\right]
\end{aligned}
$$

where $s(t)$ and $c(t)$ are abbreviations for $\sinh \left(t / T_{c}\right)$ and $\cosh \left(t / T_{c}\right)$, respectively, with $T_{c}=\sqrt{Z_{c} / g} \cdot p(t)$ and $q(t)$ represent ZMP functions on the sagittal and lateral planes, respectively. * denotes the convolution operator. The sagittal and lateral COM positions and the velocities define a WS as follows:

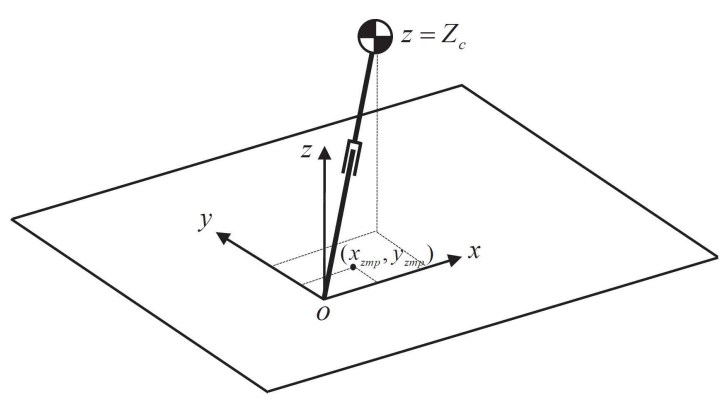

Fig. 1. 3-D LIPM.
Definition 1: A WS is defined by

$$
\begin{aligned}
& \mathbf{x}=\left[\begin{array}{ll}
x & T_{c} v
\end{array}\right]^{T} \text { for sagittal COM motion; } \\
& \mathbf{y}=\left[y T_{c} w\right]^{T} \text { for lateral COM motion }
\end{aligned}
$$

where $(x, v)$ and $(y, w)$ are the COM position and the velocity in the sagittal and lateral planes, respectively. Note that the WS is represented as a 2-D vector in each plane and that the velocity terms are multiplied by $T_{c}$. In (1), $\mathbf{x}_{i} / \mathbf{x}_{f}$ and $\mathbf{y}_{i} / \mathbf{y}_{f}$ are the initial/final WSs in the sagittal and lateral planes, respectively.

On the right-hand side of (1), the first term is the homogeneous solution component and the second term is the particular solution component of the dynamic equation of the 3-D LIPM. In the conventional 3-D LIPM [1], only the homogeneous solution component is used, which means that the ZMP remains fixed at the center of the foot polygon. Consequently, the COM motion is unmodifiable throughout the single support phase. However, the particular solution component allows the ZMP to be varied over the convex hull on the bounded foot region through the ZMP functions, $p(\mathrm{t})$ and $q(t)$; thus, it is possible to independently vary the position and the velocity of the COM throughout the single support phase. Therefore, the bipedal robot can vary its walking period, step lengths, and walking direction at every footstep in real time.

\subsection{Desired WS}

As a navigational command set for the modifiable walking pattern, the CS is defined as follows:

Definition 2: A CS is defined by

$$
\mathbf{c}=\left[\begin{array}{llllllllll}
T_{l}^{s s} & T_{l}^{d s} & S_{l} & L_{l} & \theta_{l} & T_{r}^{s s} & T_{r}^{d s} & S_{r} & L_{r} & \theta_{r}
\end{array}\right]
$$

where $T_{l / r}^{s s}, T_{l / r}^{d s}, S_{l / r}, L_{l / r}$, and $\theta_{l / r}$ are single support time, double support time, sagittal step length, lateral step length, and walking direction of the left/right swing leg.

To generate the walking pattern that satisfies the specified $\mathrm{CS}$, the conversion of the CS into the desired WS is required. Because bipedal walking is a type of periodic motion, it can be described by identifying certain particular WSs. The WSs at the end of each single support phase are identical; therefore, they are regarded as the desired WS. It is assumed that the WSs are in a steady state and that the ZMP does not vary during the conversion of the CS into the desired WS. For the conversion of the CS into the desired WS, one period of the walking configuration is considered in a steady state, as illustrated in Fig. 2. The walking configuration can be fully described by the initial or final WS for both the left and right support phases because the WS is in a steady state with no ZMP variation.

Notation 1: For the derivation, the following notations are used: 


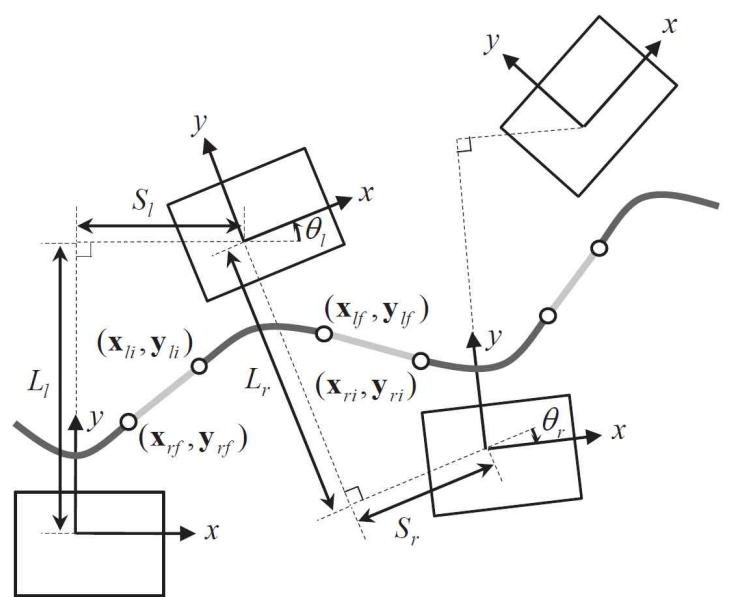

Fig. 2. Steady-state walking configuration. The rectangles represent the foot boundaries, and the thick and thin lines represent the COM trajectories in the single and double support phases, respectively. The small circles represent the initial/final COM positions in left/right support phases.

$\mathbf{x}_{l i}$ : initial sagittal WS in left support phase;

$\mathbf{x}_{l f}$ : final sagittal WS in left support phase;

$\mathbf{x}_{r i}$ : initial sagittal WS in right support phase;

$\mathbf{x}_{r f}$ : final sagittal WS in right support phase.

Note that the lateral WSs $\mathbf{y}_{l i}, \mathbf{y}_{l f}, \mathbf{y}_{r i}$, and $\mathbf{y}_{r f}$ are defined similarly.

From (1), the COM motions in the single support phase are obtained as follows:

$$
\begin{aligned}
& \mathbf{Z}_{l f}=\mathbf{A}\left(T_{l}^{s s}\right) \mathbf{Z}_{l i} \\
& \mathbf{Z}_{r f}=\mathbf{A}\left(T_{r}^{s s}\right) \mathbf{Z}_{r i}
\end{aligned}
$$

with

$$
\mathbf{Z}=\left[\begin{array}{ll}
\mathbf{x} & \mathbf{y}
\end{array}\right]
$$

During the double support phase, the COM is controlled to travel with a constant velocity, and the COM motions in the double support phase can thus be derived as follows:

$$
\begin{aligned}
& \mathbf{Z}_{l i}=\left(\mathbf{U}_{T_{r}^{d s}} \mathbf{Z}_{r f}-\mathbf{D}_{l}\right) \mathbf{R}_{\theta_{r}} \\
& \mathbf{Z}_{r i}=\left(\mathbf{U}_{T_{l}^{d s}} \mathbf{Z}_{l f}-\mathbf{D}_{r}\right) \mathbf{R}_{\theta l}
\end{aligned}
$$

with

$$
\begin{gathered}
\mathbf{R}_{\theta}=\left[\begin{array}{cc}
\cos \theta & \sin \theta \\
-\sin \theta & \cos \theta
\end{array}\right] \\
\mathbf{U}_{t}=\left[\begin{array}{cc}
1 & \frac{t}{T_{c}} \\
0 & 1
\end{array}\right], \quad \mathbf{D}_{l / r}=\left[\begin{array}{cc}
S_{l / r} & L_{l / r} \\
0 & 0
\end{array}\right] .
\end{gathered}
$$

(2) can be rewritten by substituting (3) as follows:

$$
\begin{aligned}
& \mathbf{Z}_{l f}=\mathbf{A}\left(T_{l}^{s s}\right) \mathbf{U}_{T_{r}^{d s}} \mathbf{Z}_{r f} \mathbf{R}_{\theta r}-\mathbf{A}\left(T_{l}^{s s}\right) \mathbf{D}_{l} \mathbf{R}_{\theta r} \\
& \mathbf{Z}_{r f}=\mathbf{A}\left(T_{r}^{s s}\right) \mathbf{U}_{T_{l}^{d s}} \mathbf{Z}_{l f} \mathbf{R}_{\theta l}-\mathbf{A}\left(T_{r}^{s s}\right) \mathbf{D}_{r} \mathbf{R}_{\theta l} .
\end{aligned}
$$

By utilizing the Kronecker product [17], (4) can be written in the following vectorial matrix form:

$$
\begin{aligned}
& \zeta_{l f}=\mathbf{A}_{r}^{*} \zeta_{r f}-\mathbf{b}_{r}^{*} \\
& \zeta_{r f}=\mathbf{A}_{l}^{*} \zeta_{l f}-\mathbf{b}_{l}^{*}
\end{aligned}
$$

with

$$
\begin{aligned}
& \zeta_{l / r f}=\operatorname{vec}\left(\mathbf{Z}_{l / r f}\right), \quad \mathbf{d}_{l / r}=\operatorname{vec}\left(\mathbf{D}_{l / r}\right) \\
& \mathbf{A}_{r}^{*}=\mathbf{R}_{\theta r}^{T} \otimes\left(\mathbf{A}\left(T_{l}^{s s}\right) \mathbf{U}_{T_{r}^{d s}}\right), \quad \mathbf{A}_{l}^{*}=\mathbf{R}_{\theta l}^{T} \otimes\left(\mathbf{A}\left(T_{r}^{s s}\right) \mathbf{U}_{T_{l}^{d s}}\right) \\
& \mathbf{b}_{r}^{*}=\left(\mathbf{R}_{\theta r}^{T} \otimes \mathbf{A}\left(T_{l}^{s s}\right)\right) \mathbf{d}_{l}, \quad \mathbf{b}_{l}^{*}=\left(\mathbf{R}_{\theta l}^{T} \otimes \mathbf{A}\left(T_{r}^{s s}\right)\right) \mathbf{d}_{r}
\end{aligned}
$$

where $\otimes$ denotes the Kronecker product operator. Finally, the desired WSs are obtained by solving (5), as follows:

$$
\begin{aligned}
& \zeta_{l f}=\left(\mathbf{A}_{r}^{*} \mathbf{A}_{l}^{*}-\mathbf{I}\right)^{-1}\left(\mathbf{A}_{r}^{*} \mathbf{b}_{l}^{*}+\mathbf{b}_{r}^{*}\right) \\
& \zeta_{r f}=\left(\mathbf{A}_{l}^{*} \mathbf{A}_{r}^{*}-\mathbf{I}\right)^{-1}\left(\mathbf{A}_{l}^{*} \mathbf{b}_{r}^{*}+\mathbf{b}_{l}^{*}\right)
\end{aligned}
$$

where I is the identity matrix. (6) represents the mapping relationship between the CS and the desired WS. The information from the CS is contained in $\mathbf{A}_{l / r}^{*}$ and $\mathbf{b}_{l / r}^{*}$.

After the desired WS is calculated from the CS, the ZMP variation is required to transfer the current WS to the desired WS. For this purpose, the ZMP functions, $p(t)$ and $q(t)$ are specified by constant and step functions, respectively, as follows:

$$
\begin{aligned}
& p(t)=\left\{\begin{array}{l}
P, \text { if } 0 \leq t<T \\
0, \text { otherwise }
\end{array}\right. \\
& q(t)=\left\{\begin{array}{c}
Q, \text { if } 0 \leq t<T_{s w} \\
-Q, \text { if } T_{s w} \leq t<T
\end{array}\right.
\end{aligned}
$$

where $P$ and $Q$ are the magnitudes of the constant and step functions, respectively; $T$ is the transition time from the current WS to the desired WS; and $T_{s w}$ is the switching time of the step function. The control parameters that characterize the ZMP functions, $p(t)$ and $q(t)\left(T, P, T_{s w}\right.$, and $Q)$ are solved from (1) to achieve the desired WS [4].

\section{Control Strategy for Bipedal Walking on Uneven Terrain with Unknown Height}

During bipedal walking on uneven terrain with different heights, the contact of the swing leg with the terrain causes a disturbance between the robot and the terrain. To 


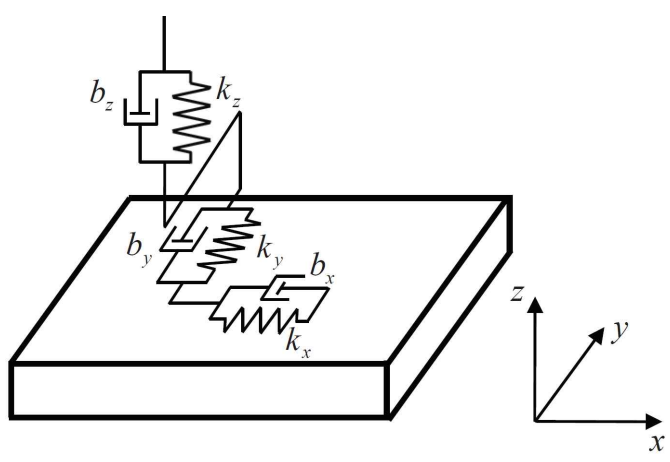

Fig. 3. Virtual spring-damper model for the foot.

compensate for this disturbance, virtual spring-damper model for the foot is proposed, as illustrated in Fig. 3. This model comprises three virtual spring-damper units. One linear spring-damper unit moves in the direction of the $z$ axis, and there are two rotational spring-damper units, one moving in the direction of the $x$-axis and one in the direction of the $y$-axis. Based on the torques and ground reaction force that are measured by the FSRs on each foot, displacements are generated using the following equations:

$$
\begin{aligned}
& T_{x}=k_{x} \theta_{x}+b_{x} \dot{\theta}_{x} \\
& T_{y}=k_{y} \theta_{y}+b_{y} \dot{\theta}_{y} \\
& F_{z}=k_{z} d_{z}+b_{z} \dot{d}_{z}
\end{aligned}
$$

where $T_{x}, T_{y}$, and $F_{z}$ are the two torques and the ground reaction force on the foot, respectively. $k_{x}, k_{y}$, and $k_{z}$ are the spring coefficients, and $b_{x}, b_{y}$, and $b_{z}$ are the damper coefficients. $\theta_{x} / \dot{\theta}_{x}$ and $\theta_{y} / \dot{\theta}_{y}$ are the displacements /velocities of the rotational spring-damper units, and $d_{z} / \dot{d}_{z}$ is the displacement/velocity of the linear springdamper unit. Subsequently, the position and orientation of the foot are modified, under the assumption that physical spring-damper units are actually installed. Note that each virtual spring-damper unit moves only in its given operational direction.

Fig. 4 depicts the bipedal walking on uneven terrain with an unknown height. In the single support phase, the sagittal and lateral foot trajectories of the swing leg, $\left(x_{\text {foot }}(t), y_{\text {foot }}(t)\right)$ are obtained via cubic spline interpolation as follows:

$$
\begin{aligned}
& x_{f o o t}(t)=-2 \frac{S_{l / r}+S_{r / l}^{p r e}}{\Delta T^{3}} t^{3}+3 \frac{S_{l / r}+S_{r / l}^{p r e}}{\Delta T^{2}} t^{2}-S_{r / l}^{p r e} \\
& y_{f o o t}(t)=-2 \frac{L_{l / r}+L_{r / l}^{p r e}}{\Delta T^{3}} t^{3}+3 \frac{L_{l / r}+L_{r / l}^{p r e}}{\Delta T^{2}} t^{2}-L_{r / l}^{p r e} \\
& \Delta T=T_{l / r}^{s s}
\end{aligned}
$$

where $S_{r / l}^{p r e}$ and $L_{r / l}^{p r e}$ represent the sagittal and lateral step lengths, respectively, of the previous footstep. The vertical foot trajectory of the swing leg, $z_{\text {foot }}(t)$, is obtained via a cycloid function, and an additional trajectory $\Delta z_{\text {foot }}(t)$ is added to the vertical foot trajectory depending on the

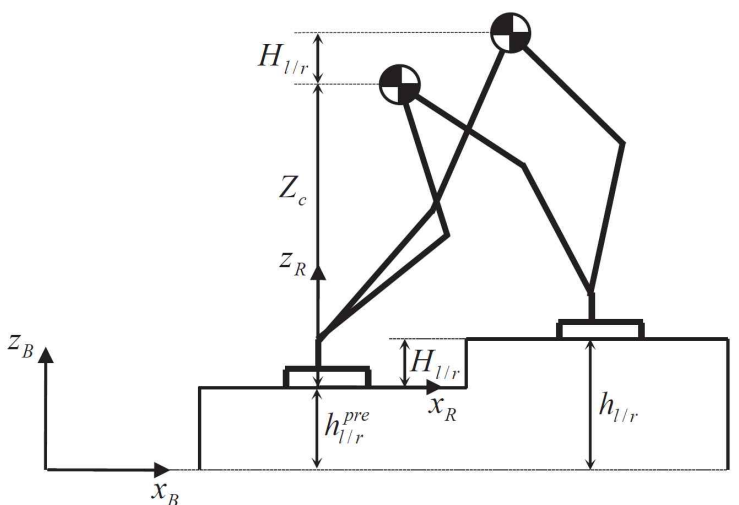

Fig. 4. Bipedal walking on uneven terrain with unknown height.

foot height $H_{l / r}$. This additional trajectory is generated via cubic spline interpolation as follows:

$$
z_{\text {foot }}(t)=R\left\{1-\cos \left(\frac{2 \pi t}{T_{l / r}^{s s}}\right)\right\}+\Delta z_{\text {foot }}(t)
$$

with

$$
\begin{aligned}
& \Delta z_{f o o t}(t)=-2 \frac{H_{l / r}+H_{r / l}^{p r e}}{\Delta T^{3}} t^{3}+3 \frac{H_{l / r}+H_{r / l}^{p r e}}{\Delta T^{2}} t^{2}-H_{r / l}^{p r e} \\
& \Delta T=T_{l / r}^{s s}
\end{aligned}
$$

where $H_{r / l}^{\text {pre }}$ is the foot height of the swing leg from the previous footstep. $R$ denotes the radius of the cycloid circle. In eqs. (9) and (10), the initial and final velocities for the cubic spline interpolation were set to zero. Consequently, the stable foot motion is able to be achieved by the foot trajectories with the zero initial and final velocities.

If the sole of the swing leg contacts the uneven terrain with an unknown height at $t=t_{c}$, then the foot height $H_{l / r}$ is modified as follows:

$$
H_{l / r}=z_{f o o t}\left(t_{c}\right)+d_{z} .
$$

The height of the terrain is estimated by $z_{\text {foot }}\left(t_{c}\right)$ using the FSRs on the each foot and the disturbance caused by the unexpected contact is compensated by $d_{z}$ using the virtual spring-damper controllers. Then, the commanded foot height for the next footstep, $H_{l / r}^{\text {next }}$, is specified as follows:

$$
H_{l / r}^{\text {next }}=-h_{l / r}
$$

with

$$
h_{l / r}=h_{l / r}^{p r e}+H_{l / r}
$$

Note that $H_{l / r}$ and $H_{l / r}^{n e x t}$ are defined with respect to the local coordinate frame of the support leg, and $h_{l / r}$ and $h_{l / r}^{\text {pre }}$ are defined with respect to the base coordinate frame of the 
ground, as illustrated in Fig. 4.

In the double support phase, the COM height does not remain constant, as in the conventional 3-D LIPM; instead, the vertical COM trajectory is modified to match the estimated foot height of the swing leg, $H_{l / r}$, from the single support phase. As illustrated in Fig. 4, the COM height preserves a constant $Z_{c}$ during the single support phase, and it then moves to $Z_{c}+H_{l / r}$ during the double support phase through a cubic spline interpolation as follows:

$$
\begin{aligned}
& z(t)=-2 \frac{H_{l / r}}{\Delta T^{3}} t^{3}+3 \frac{H_{l / r}}{\Delta T^{2}} t^{2}+Z_{c} . \\
& \Delta T=T_{l / r}^{d s}
\end{aligned}
$$

Note that $z(t)$ is defined with respect to the local coordinate frame of the support leg. In Eq. (13), the initial and final velocities for the cubic spline interpolation were set to zero. Consequently, the stable COM motion is able to be achieved by the vertical COM trajectory with the zero initial and final velocities.

The overall procedure for the proposed walking pattern generation method is summarized in Algorithm 1. The CS, which is predefined or provided by the footstep planner [18-20], is entered as an input to the proposed walking pattern generator at every sample time. In the single support phase, the CS is converted into the desired WS using (6), and the control parameters of the ZMP functions are then calculated for the ZMP variation. Using these control parameters, the sagittal and lateral COM trajectories are generated using (1). The foot trajectory of the swing leg is generated using (9), (10). If the sole of the swing leg contacts the terrain, the foot height is modified using (11), and the foot height for the next footstep is determined using (12). In the double support phase, the vertical COM trajectory is generated using (10). Subsequently, the leg joint trajectories of the robot are calculated using inverse kinematics, and these steps are repeated until the walking motion is terminated.

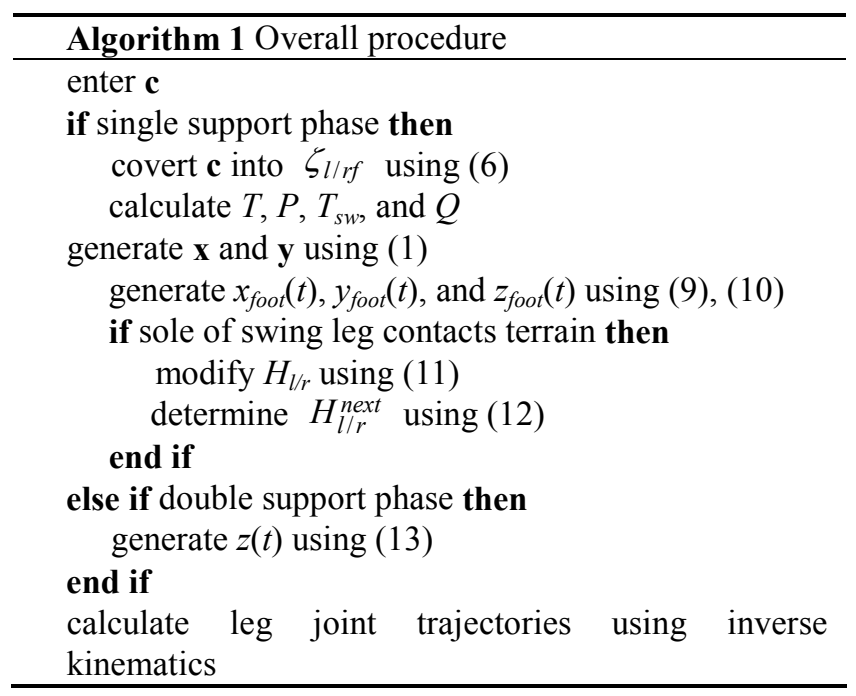

\section{Computer Simulation}

\subsection{Robot model}

To verify the performance of the proposed method, we used a simulated model of the small-sized humanoid platform DARwin-OP, depicted in Fig. 5(a). The height and weight of this platform are $45.5 \mathrm{~cm}$ and $2.8 \mathrm{~kg}$, respectively. The simulated model of DARwIn-OP was produced using Webots, which is a 3-D robotics simulation software that enables users to perform physical and dynamical simulations; the model is depicted in Fig. 5(b).

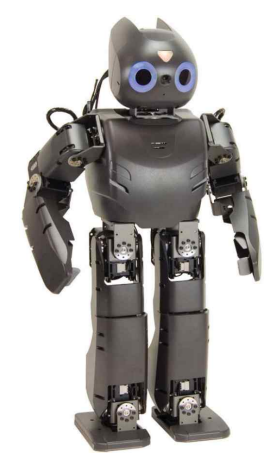

(a)

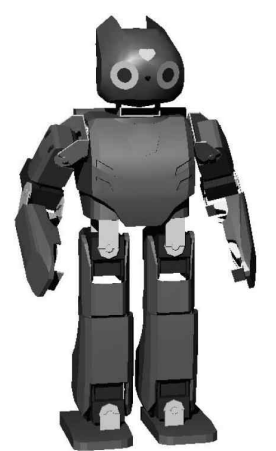

(b)
Fig. 5. (a) DARwIn-OP; (b) Simulation model.

\subsection{Simulation results}

Five boards with different heights $(0.5,1.0$, and $1.5 \mathrm{~cm})$, sizes, and directions were placed in the simulation environment. Tables 1 presents the parameter settings for

Table 1. Virtual spring-damper model coefficients

\begin{tabular}{c|c|c}
\hline$x$-axis virtual spring-damper & $k_{x}$ & 1000 \\
\cline { 2 - 3 } model & $b_{x}$ & 100 \\
\hline \multirow{2}{*}{$\begin{array}{c}\text { y-axis virtual spring-damper } \\
\text { model }\end{array}$} & $k_{y}$ & 1000 \\
\cline { 2 - 3 } $\begin{array}{c}z \text {-axis virtual spring-damper } \\
\text { model }\end{array}$ & $b_{y}$ & 100 \\
\cline { 2 - 3 } & $k_{z}$ & 100 \\
\hline
\end{tabular}

Table 2. CS list and estimated foot heights (time, length, and angle are expressed in units of seconds, centimeters, and degrees, respectively.)

\begin{tabular}{c|c|c|c|c|c|c|c|c|c|c|c|c}
\hline \multirow{2}{*}{ Steps } & \multicolumn{10}{|c|}{ CS list } \\
\cline { 2 - 13 } & $T_{l}^{s s}$ & $T_{l}^{d s}$ & $S_{l}$ & $L_{l}$ & $\theta_{l}$ & $T_{r}^{s s}$ & $T_{r}^{d s}$ & $S_{r}$ & $L_{r}$ & $\theta_{r}$ & $H_{l}$ & $H_{r}$ \\
\hline $1^{\text {st }}$ & 0.8 & 0.4 & 7.0 & 7.4 & 0.0 & 0.8 & 0.4 & 7.0 & -7.4 & 0.0 & 0.00 & 0.00 \\
\hline $2^{\text {nd }}$ & 0.8 & 0.4 & 7.0 & 7.4 & 0.0 & 0.8 & 0.4 & 7.0 & -7.4 & 0.0 & 0.97 & 0.0 \\
\hline $3^{\text {rd }}$ & 0.8 & 0.4 & 5.0 & 7.4 & 0.0 & 0.8 & 0.4 & 5.0 & -7.4 & 0.0 & 0.0 & -0.46 \\
\hline $4^{\text {th }}$ & 0.8 & 0.4 & 7.0 & 7.4 & 0.0 & 0.8 & 0.4 & 7.0 & -7.4 & 0.0 & 0.45 & 0.0 \\
\hline $5^{\text {th }}$ & 0.8 & 0.4 & 7.0 & 7.4 & 0.0 & 0.8 & 0.4 & 7.0 & -10.4 & 0.0 & 0.0 & -0.93 \\
\hline $6^{\text {th }}$ & 0.8 & 0.4 & 9.0 & 7.4 & 0.0 & 0.8 & 0.4 & 9.0 & -7.4 & 0.0 & 1.44 & 0.0 \\
\hline $7^{\text {th }}$ & 0.8 & 0.4 & 7.0 & 7.4 & 0.0 & 0.8 & 0.4 & 7.0 & -7.4 & 0.0 & 0.0 & -0.43 \\
\hline $8^{\text {th }}$ & 0.8 & 0.4 & 8.0 & 8.4 & 20.0 & 0.8 & 0.4 & 8.0 & -7.4 & 20.0 & -0.48 & 0.0 \\
\hline $9^{\text {th }}$ & 0.8 & 0.4 & 7.0 & 7.4 & 0.0 & 0.8 & 0.4 & 7.0 & -7.4 & 0.0 & 0.0 & -0.47 \\
\hline $10^{\text {th }}$ & 0.8 & 0.4 & 7.0 & 7.4 & 0.0 & 0.8 & 0.4 & 7.0 & -7.4 & 0.0 & 0.0 & 0.0 \\
\hline $11^{\text {th }}$ & 0.8 & 0.4 & 0.0 & 7.4 & 0.0 & 0.8 & 0.4 & 0.0 & -7.4 & 0.0 & 0.0 & 0.0 \\
\hline & & & & & & & & & & & &
\end{tabular}



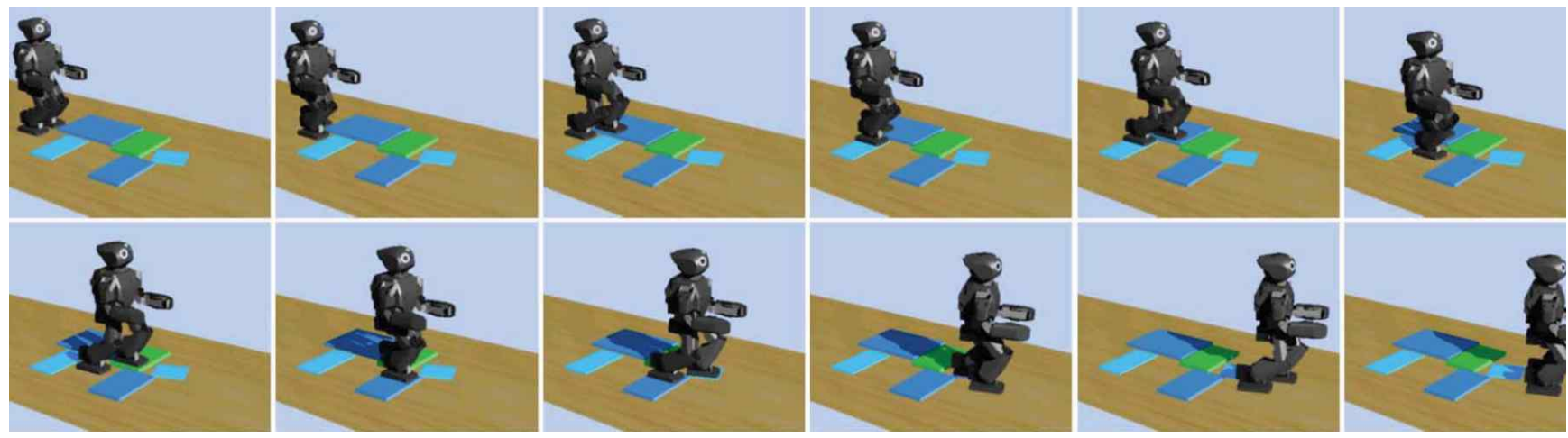

Fig. 6. Snapshots of the walking simulation result (left to right, top to bottom). The heights of the boards were set to 0.5 , 1.0 , and $1.5 \mathrm{~cm}$.
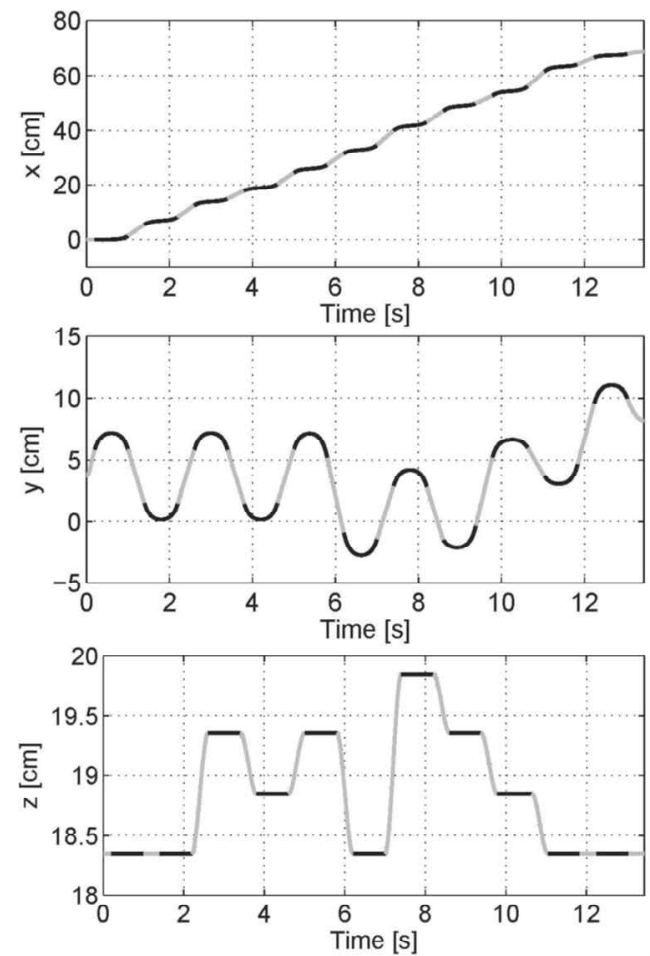

Fig. 7. Sagittal, lateral, and vertical COM trajectories with respect to the base coordinate frame of the ground. The thick and thin lines represent the COM trajectories in the single and double support phases, respectively.

the virtual spring-damper models.

Fig. 6 presents snapshots of the walking simulation result. The bipedal robot successfully walked on the uneven terrain of unknown height modifying independently the sagittal and lateral step lengths, and the walking direction at every footstep. Fig. 7 presents the generated COM trajectories with respect to the base coordinate frame of the ground. The sagittal and lateral COM trajectories were obtained to achieve modifiable bipedal walking that satisfied the CS list presented in Table 2. In addition, the heights of the boards were estimated by using the proposed method. Then, the foot height $H_{l / r}$ was modified for walking on the uneven terrain with an unknown height as shown in Table 2. The vertical COM trajectory maintained a constant height during the single support phase. Then, it moved up or down depending on the estimated foot heights during the double support phase. The ZMP trajectories during the modifiable bipedal walking in the simulation were measured and are presented in Fig. 8. The ZMP trajectories along the $x$-axis and the $y$-axis followed the foot trajectories, with slight variations. The primary reasons for these slight variations in the ZMP trajectories were the disturbance caused by the contact between the robot's foot and the terrain at different heights and the differences in dynamics between the robot and the 3-D LIPM. However, the proposed virtual spring-damper model allowed for compensation of these disturbances and dynamics differences, and it was observed that the ZMP trajectories fell within the boundaries of the foot trajectories during walking. Consequently, the robot could walk on an uneven terrain with an unknown height while maintaining its stability. In addition, the walking simulations on the boards with various heights were performed. As a result, the allowable height of the board was $4.0 \mathrm{~cm}$, which is about $20.0 \%$ of the overall leg length of the DarwIn-OP. Although the height of the board was well estimated, the allowable height of the board was limited. It is because the humanoid is unable to walk stably on the board with the high height by the present COM trajectory generation method.

\section{Conclusion}

This paper proposed a walking pattern generator for modifiable bipedal walking on uneven terrain with an unknown height. A 3-D LIPM was used as a simplified model of a bipedal robot, and the sagittal and lateral COM motions were obtained from the dynamic equation of the 3D LIPM. By allowing for variations in the ZMP, it became possible to vary the COM motions throughout the single support phase. The CS was defined and then converted into the desired WS. Then, the control parameters of the ZMP 

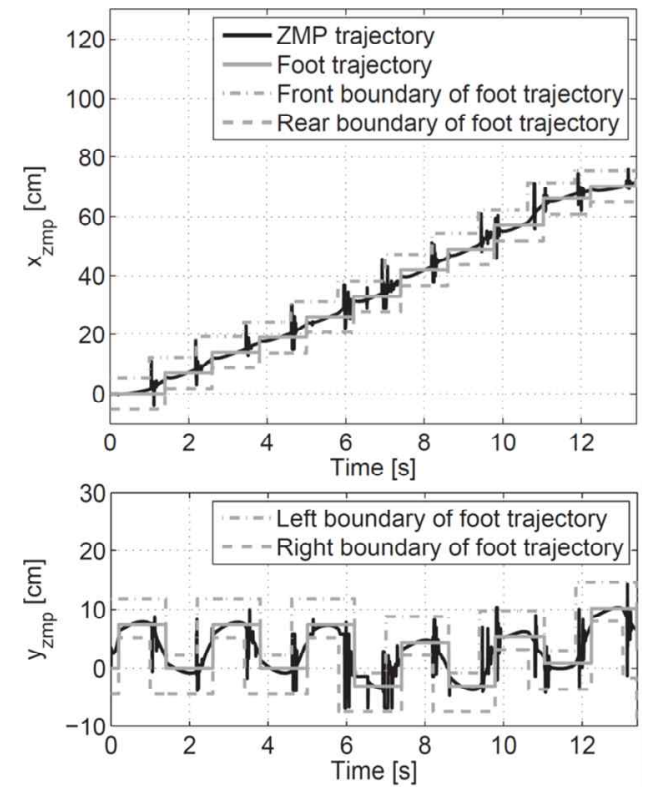

Fig. 8. ZMP trajectories measured in the walking simulation.

functions were obtained. In addition, when the swing leg came into contact with the terrain, the disturbance between the robot and the terrain was compensated by the virtual spring-damper model of the foot, and the foot and vertical COM trajectories were modified depending on the estimated foot height at that moment. Consequently, using the proposed method, the bipedal robot was able to stably walk on uneven terrain without being supplied any information regarding the height of the terrain by modifying its walking periods, step lengths, and walking direction at every footstep.

\section{References}

[1] S. Kajita, F. Kanehiro, K. Kaneko, K. Fujiwara, K. Yokoi, and H. Hirukawa, "A realtime pattern generator for biped walking," in Proc. IEEE Int. Conf. Robot. Autom., 2002, vol. 1, pp. 31-37.

[2] T. Aoyama, Y. Hasegawa, K. Sekiyama, and T. Fukuda, "Stabilizing and direction control of efficient 3-D biped walking based on PDAC," IEEE/ASME Trans. Mechatron., vol. 14, no. 6, pp. 712- 718, Dec. 2009.

[3] K. Harada, S. Hattori, H. Hirukawa, M. Morisawa, S. Kajita, and E. Yoshida, "Two-stage time-parametrized gait planning for humanoid robots," IEEE/ASME Trans. Mechatron., vol. 15, no. 5, pp. 694-703, Oct. 2010.

[4] Y.-D. Hong, B.-J. Lee, and J.-H. Kim, "Command state-based modifiable walking pattern generation on an inclined plane in pitch and roll directions for humanoid robots," IEEE/ASME Trans. Mechatron., vol. 16, no. 4, pp. 783-789, Aug. 2011.

[5] Y.-D. Hong, C.-S. Park, and J.-H. Kim, "Stable bipedal walking with a vertical center of mass motion by an evolutionary optimized central pattern generator," IEEE Trans. Ind. Electron., vol. 61, no. 5, pp. 22462355, May 2014.

[6] Y.-D. Hong, “An evolutionary optimization approach for optimal hopping of humanoid robots," J. Elect. Eng. Technol., vol. 10, no. 6, pp. 2420-2426, Nov. 2015.

[7] S. Kajita and K. Tani, "Study of dynamic biped locomotion on rugged terrain -Derivation and application of the linear inverted pendulum mode-," in Proc. IEEE Int. Conf. Robot. Autom., 1991, pp. 1405-1411.

[8] Q. Huang and Y. Nakamura, "Sensory reflex control for humanoid walking," IEEE Trans. Robot., vol. 21, no. 5, pp. 977-984, Oct. 2005.

[9] S. Kajita, M. Morisawa, K. Harada, K. Kaneko, F. Kanehiro, K. Fujiwara, and H. Hirukawa, "Biped walking pattern generator allowing auxiliary ZMP control," in Proc. IEEE/RSJ Int. Conf. Intell. Robot. Syst., 2006, pp. 2993-2999.

[10] K. Nishiwaki and S. Kagami, "Walking control on uneven terrain with short cycle pattern generation," in Proc. IEEE-RAS Int. Conf. Humanoid Robots, 2007, pp. 447-453.

[11] T. Takubo, Y. Imada, K. Ohara, Y. Mae, and T. Arai, "Rough terrain walking for bipedal robot by using ZMP criteria map," in Proc. IEEE Int. Conf. Robot. Autom., 2009, pp. 788-793.

[12] Y. Zheng, M. C. Lin, D. Manocha, A. H. Adiwahono, and C.-M. Chew, "A walking pattern generator for biped robots on uneven terrains," in Proc. IEEE/RSJ Int. Conf. Intell. Robot. Syst., 2010, pp. 4483-4488.

[13] E. Ohashi, T. Sato, and K. Ohnishi, "A walking stabilization method based on environmental modes on each foot for biped robot," IEEE Trans. Ind. Electron., vol. 56, no. 10, pp. 3964-3974, Oct. 2009.

[14] H.-J. Kang, K. Hashimoto, H. Kondo, K. Hattori, K. Nishikawa, Y. Hama, H.-O. Lim, A. Takanishi, K. Suga, and K. Kato, "Realization of biped walking on uneven terrain by new foot mechanism capable of detecting ground surface," in Proc. IEEE Int. Conf. Robot. Autom., 2010, pp. 5167-5172.

[15] Y.-D. Hong and J.-H. Kim, "3-D command statebased modifiable bipedal walking on uneven terrain," IEEE/ASME Trans. Mechatron., vol. 18, no. 2, pp. 657-663, Apr. 2013.

[16] Y.-D. Hong and B.-J. Lee, "Experimental study on modifiable walking pattern generation for handling infeasible navigational commands," J. Elect. Eng. Technol., vol. 10, no. 6, pp. 2368-2375, Nov. 2015.

[17] H. V. Henderson and S. R. Searle, "The vecpermutation matrix, the vec operator and kronecker products: a review," Linear Multilinear Algebra, vol. 9, no. 4, pp. 271-288, 1981.

[18] Y.-D. Hong, Y.-H. Kim, J.-H. Han, J.-K. Yoo, and J.H. Kim, "Evolutionary multiobjective footstep 
planning for humanoid robots," IEEE Trans. Syst. Man. Cybern. C, Appl. Rev., vol. 41, no. 4, pp. 520532, Jul. 2011.

[19] Y.-D. Hong and J.-H. Kim, "An evolutionary optimized footstep planner for the navigation of humanoid robots," Int. J. Humanoid Robot., vol. 9, no. 1, Mar. 2012.

[20] Y.-D. Hong, "Real-time footstep planning and following for navigation of humanoid robots," $J$. Elect. Eng. Technol., vol. 10, no. 5, pp. 2142-2148, Sep. 2015.

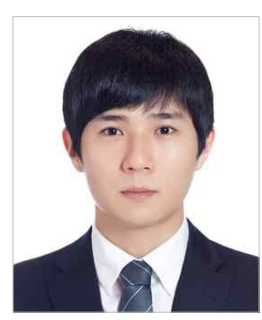

Young-Dae Hong received the B.S., M.S., and Ph.D. degrees in electrical engineering from KAIST, Daejeon, Korea, in 2007, 2009, and 2013 respectively. Since 2014, he has been with the department of electrical and computer engineering, Ajou University, Suwon, Korea, where he is currently an Assistant Professor. His current research interests include humanoid robotics, especially in bipedal walking pattern generation, footstep planning, multi / single objective evolutionary optimization, and biologically inspired control.

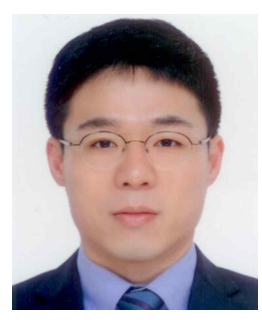

Ki-Baek Lee received the B.S. and Ph.D. degrees in electrical engineering from KAIST, Daejeon, Korea, in 2005 and 2014, respectively. Since 2014, he has been an Assitant Professor with the Department of Electrical Engineering, Kwangwoon University, Seoul, Korea. He has researched computational intelligence, in particular, in the area of swarm intelligence and multi-objective evolutionary algorithms (MOEAs). His research interests also include a real world application such as on-line multi-objective evolutionary navigation for humanoid robots. 